\title{
СИСТЕМА УПРАВЛЕНИЯ, ОБЕСПЕЧИВАЮЩАЯ ЭНЕРГОЭФФЕКТИВНОЕ ФУНКЦИОНИРОВАНИЕ ХОЛОДИЛЬНОЙ ТУРБОКОМПРЕССОРНОЙ УСТАНОВКИ
}

\author{
Гурский А.А. ${ }^{1}$, Денисенко В.А. ${ }^{2}$, Гончаренко А.Е. ${ }^{2}$ \\ ${ }^{1}$ Одесская национальная академия пищевых технологий, г. Одесса \\ ORCID: $\underline{0000-0001-5158-2125}$ \\ ${ }^{2}$ Одесская национальная академия пищевых технологий, г. Одесса
}

Copyright $(\mathcal{C} 2014$ by author and the journal "Automation technological and business - processes". This work is licensed under the Creative Commons Attribution International License (CC BY). http://creativecommons.org/licenses/by/4.0/

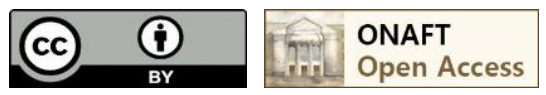

DOI: $10.15673 / 2312-3125$.

\begin{abstract}
Аннотация
В статье рассматривается система управления, обеспечивающая регулирование производительности и энергоэффективное функционирование холодильной турбокомпрессорной установки. Представлен обзор современных достижений в соответствующей области. Поставлена цель работы - повышение энергоэффективности функционирования установки, состоящей из турбокомпрессора и конденсатора. Для достижения поставленной цели необхоимо было разработать модель объекта управления, представляющую холодильную установку с центробежным компрессором, а затем выполнить синтез соответствующих систем управления и анализ их функционирования. В настоящей работе отражается особенность функционирования предложенной системы энергоэффективного управления объектом турбокомпрессор-конденсатор. Описывается принцип координирующего управления установкой, использования известной информации о траектории дрейфа экстремума, а также применения автоматического оптимизатора в координирующей системе для поиска экстремума или для проверки и корректировки информации о траектории дрейфа экстремума. Представляется многоуровневый закон управления предложенной системы, который обеспечивает необходимое соотношение параметров установки, как при переходных, так и при стационарных режимах для обеспечения координирующего управления a, следовательно, и для энергоэффективного функционирования турбокомпрессорной установки. Представлены результаты моделирования типовой САУ турбокомпрессорной установкой и САУ построенной на принципе координирующего управления. На основании анализа результатов моделирования и особенностей функционирования различных систем делается вывод о том, что предлагаемая система управления имеет ряд отличительных достоинств, которые подтверждают принципиальную пригодность ее для практического использования.
\end{abstract}

\section{Abstract}

The control system providing for the effective functioning of the turbo compressor machine is considered in the article. This system provides for regulation of the refrigeration turbo compressor machine capacity. The review of modern achievements in corresponding field is presented. The aim of our research work is to improve the effective 
functioning of the centrifugal compressor machine with the condenser. The control system designing of the turbo compressor machine was worked out in this work. The control system has to provide for regulation of turbo compressor capacity with by means of two controlling actions. The object control of the offered system functioning the principle is described in the present work. The coordinating control of refrigeration turbo compressor machine the principle is determined. The use of the known information about the extremum drift trajectory the principle show. The feature of the automatic optimizer application in the coordinating system is considered. The feature of the automatic optimizer application for the information correction about the extremum drift trajectory is shown. The multilevel law of the offered effective system control is represented. This coordinating control system provides for the necessary proportion of the machine parameters, both the dynamic and the static models. This proportion is provided for the effective functioning of the turbo compressor machine. The different automatic control systems of the turbo compressor machine modelling results for the comparison are presented in this article. The typical automatic control systems of the centrifugal compressor machine modeling results are presented. The offered coordination control system of the refrigeration machine with the centrifugal compressor modeilling results are presented in this article. It is quite possible for us to apply the present control system of the turbo compressor machine in the future.

\section{Ключевые слова}

Холодильная установка, центробежный компрессор, координирующее управление, поиск экстремума.

Введение

Известно, что энергоэффективное функционирование центробежной компрессорной установки можно обеспечить за счет регулирования производительности ее посредством использования одновременно двух управляющих воздействий [1 - 4]. В отдельной литературе такое регулирование производительности получило название - комбинированное [1]. В настоящее время в некоторых установках с компрессорами динамического принципа действия применяется данное комбинированное регулирование производительности, которое дает наименьшую потребляемую мощность привода компрессора в основном для статического режима работы [5].

Обзор современных достижений

Регулирование производительности центробежного компрессора (ЦБК) посредством использования двух управляющих воздействий можно реализовать в рамках поисковой системы экстремального регулирования. В работе [6] приведено описание такой системы, реализующей комбинированное регулирование производительности ЦБК. Такая система, представляет собой состав традиционной (типовой) САУ и одноканальной (однопараметрической) системы оптимизации статических режимов [6, 7]. Данная система имеет ряд недостатков, а именно, движение к экстремуму осуществляет после переходного процесса, связанного с регулированием производительности ЦБК, и с присутствием постоянных пробных движений, при этом, не использует известную информацию о траектории дрейфа экстремума. В работе [8] была предложена другая система, которая построена на принципе координирующего управления [9] и с использованием автоматического оптимизатора. Данные системы, сравнительный анализ которых приведен в работе [7], рассматриваются для обеспечения регулирования производительности центробежного компрессора посредством изменения скорости вращения вала $n_{v}$ и угла установки лопаток диффузора $\alpha_{\text {Д. }}$

В некоторых ЦБК, в том числе малорасходных (в связи с конструктивными затруднениями), поворотные лопатки диффузора или лопатки входного регулирующего аппарата (ВРА) не предусмотрены [10]. Таким образом, в настоящей работе приводится исследование касающиеся целесообразности применения предложенной системы $[7,8]$, построенной на принципе координирующего управления [9], для турбокомпрессорной установки у которой отсутствуют поворотные лопатки диффузора или ВРА.

Цель и задача настоящей работы

Цель настоящей работы - повышение энергоэффективности функционирования установки, состоящей из турбокомпрессора и конденсатора.

Для достижения поставленной цели необходимо было разработать модель объекта управления, представляющую холодильную установку с центробежным компрессором, а затем выполнить синтез соответствующих систем управления и анализ их функционирования (качества регулирования и обеспечения наименьшего энергопотребления). 


\section{4 ТЕХНІЧНІ ЗАСОБИ ТА ІНФОРМАЦІЙНІ ТЕХНОЛОГІЇ В СИСТЕМАХ УПРАВЛІННЯ}

Особенность разработанной системы

В настоящей работе разрабатываемая система управления должна обеспечить наименьшую суммарную потребляемую мощность приводов установки $\Sigma P$, а именно, привода центробежного компрессора и привода системы охлаждения конденсатора. Если учесть, что холодопроизводительность машины зависит от скорости вращения вала привода ЦБК $n_{v}$ и вала привода системы охлаждения конденсатора $n_{k}[10]$, то возникает вопрос, какое оптимальное соотношение должно быть между скоростями вращения $n_{v}$ и $n_{k}$ валов приводов установки, при требуемой холодопроизводительности, чтобы суммарная потребляемая мощность $\Sigma P$ была бы минимальной?

Данное оптимальное соотношение между $n_{v}$ и $n_{k}$ формируется в системе при отработке оптимального соотношения технологических параметров между производительностью ЦБК $Q$ и давлениями нагнетания $P_{K}$ и всасывания $P_{0}$.

Оптимальное соотношение между $Q, P_{K}$ и $P_{O}$ можно определить в результате анализа характеристик турбокомпрессорной установки, до функционирования системы управления, или в результате работы автоматического оптимизатора, обеспечивающего поиск экстремума показателя качества, в процессе функционирования системы, путем ввода поисковых - тестовых воздействий (рисунок 1).

Представленная на рисунке 1 структурная схема системы управления, аналогична предложенной ранее в работах [7, 8]. Однако в настоящей работе данная система не связана с поворотными лопатками диффузора ЦБК, так как в рассматриваемом компрессоре они отсутствуют.

Рассматриваемая система управления (рисунок 1) имеет отличительную особенность - она использует известную информацию о траектории дрейфа экстремума, представленную в виде соотношения между $Q, P_{K}$ и $P_{0}$. Такая система строится на принципах координирующего управления. Координирующее управление связано с режимом разделения движения [9] согласно которому отрабатывается невязка $\varphi$ по соотношению параметров $(Q$, $P_{K}$ и $\left.P_{O}\right)$ при переходном процессе, связанном с регулированием производительности ЦБК, тем самым, обеспечивается движение к экстремуму показателя качества $P$ начиная с начала переходного процесса. При этом автоматический оптимизатор изменяет непосредственно не задающее воздействие (как в системе описанной в работе [6]), а коэффициент соотношения между $Q, P_{K}$ и $P_{0}$, после переходного процесса связанного с регулированием производительности ЦБК. В данном случае можно отметить, что автоматический оптимизатор необходим для проверки известной информации о траектории дрейфа экстремума или для проверки правильности выхода системы в точку экстремума показателя качества $P$.

Закон управления системы

Закон управления системы, структурная схема которой представлена на рисунке 1, двухуровневый. Он имеет следующий вид:

$$
u=u_{q}+\alpha_{\kappa} \cdot u_{p}=\left[\begin{array}{ll}
u_{1 G_{w V}} & u_{2 n_{v}}
\end{array}\right]^{T}
$$

где $u_{q}$ - стабилизирующий вектор управления, обусловливающий движение к целевому многообразию определяемому соотношением $Q, P_{K}$ и $P_{0}$;

$u_{p}$ - варьирующий вектор, обусловливающий движение по целевому многообразию;

$\alpha_{\kappa}-$ коэффициент веса второго уровня - параметр, определяющий структурно-временную соподчиненность процесса (режим разделения движения - первоначальное движение к целевому многообразию регулируемого соотношения, а затем по нему).

Закон управления первого уровня взаимосвязанный (координирующий), он связан с отработкой невязки $\varphi$ оптимального соотношения, определяющего наименьшее значение потребляемой мощности приводов установки. Закон управления первого уровня можно представить в следующем виде:

$$
\bar{u}_{q}=\varphi \cdot\left[\begin{array}{c}
\left(c_{01}+c_{11} / P\right) \\
\left(c_{02}+c_{12} / P\right)
\end{array}\right]=\left[\begin{array}{c}
{ }^{<1>} u_{1} \\
{ }^{<2>} u_{2}
\end{array}\right],
$$

где $\mathrm{c}_{01} \mathrm{c}_{11} \mathrm{c}_{02} \mathrm{c}_{13}$ - параметры настройки регуляторов 1-го уровня;

$\varphi=f\left(P_{k}, P_{0}, G_{x . a}\right)$ - невязка по соотношению параметров, при этом, расход холодильного агента $G_{x . a}$ (производительность ЦБК $Q$ ) при отсутствии средства измерения может быть определен на основе известных параметров, то есть $G_{x . a}=f\left(P_{k}, P_{0}, n_{v}\right)$. 


\section{4 ТЕХНІЧНІ ЗАСОБИ ТА ІНФОРМАЦІЙНІ ТЕХНОЛОГІЇ В СИСТЕМАХ УПРАВЛІННЯ}

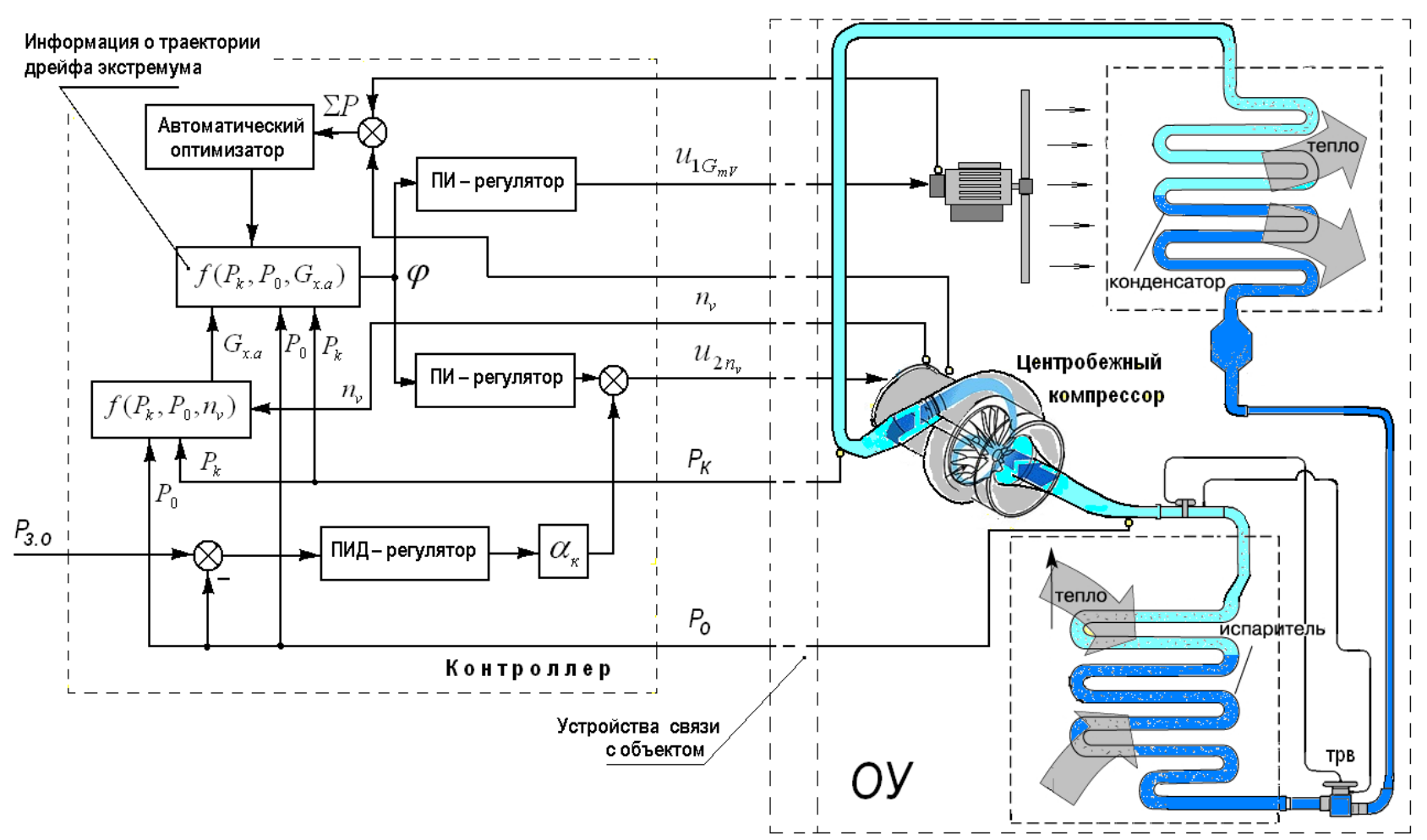

Рис.1 - Структурно-технологическая схема, отражающая принцип управления холодильной турбокомпрессорной установкой

Закон 2-го уровня управления, необходимый для отработки ошибки регулирования, следующий:

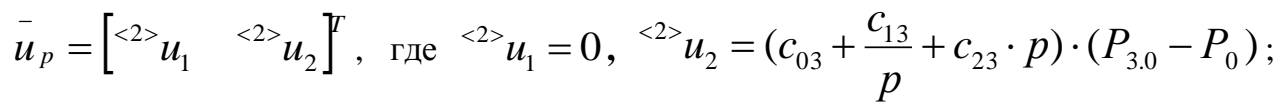

где $c_{03}, c_{13}, c_{23}-$ параметры настройки ПИД-регулятора;

$T$-знак транспонирования;

$P_{0}, P_{3,0}-$ фактическое и заданное давления всасывания (кипения).

Анализ результатов моделирования

Вышеприведенный закон управления был реализован для холодильной турбокомпрессорной установки, представленной в качестве модели в рамках виртуального стенда. Данный виртуальный стенд был разработан в программной среде Labview для проведения различных экспериментов в области анализа функционирования холодильной турбокомпрессорной установки при различных системах управления [11]. В результате экспериментов были получены графики переходных процессов, на основании которых можно сделать вывод о целесообразности применения предложенной системы с координирующим законом управления.

Из графиков, представленных на рисунке 2 видно, что в отличие от типовой САУ холодильной установкой, предлагаемая система обеспечивает практически нулевые отклонения от соотношения параметров при изменении производительности установки на 35 \%. При этом, время переходных процессов при типовой (одноконтурной) системе управления и при координирующей практически равно, также как и перерегулирования. 


\section{4 ТЕХНІЧНІ ЗАСОБИ ТА ІНФОРМАЦІЙНІ ТЕХНОЛОГІЇ В СИСТЕМАХ УПРАВЛІННЯ}

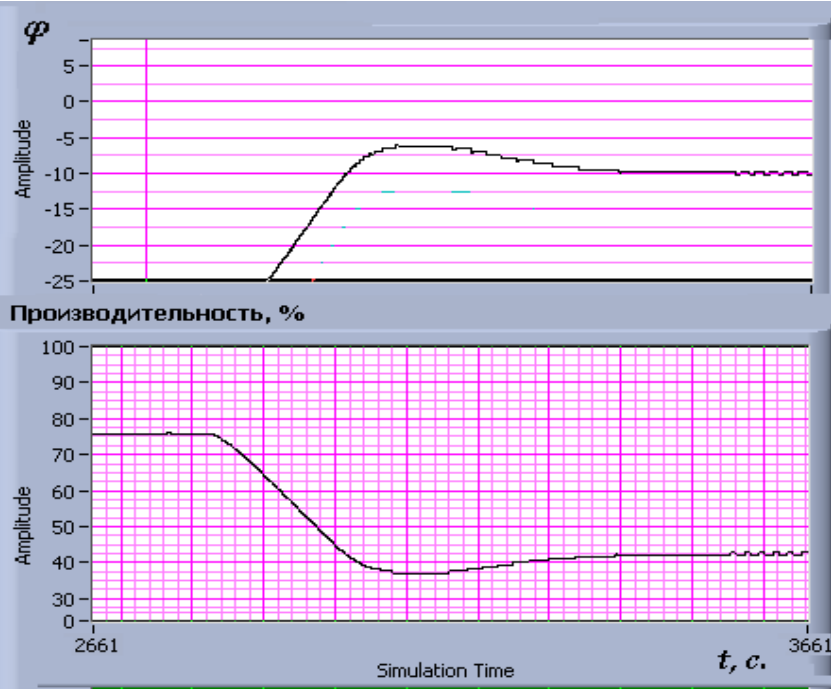

a)

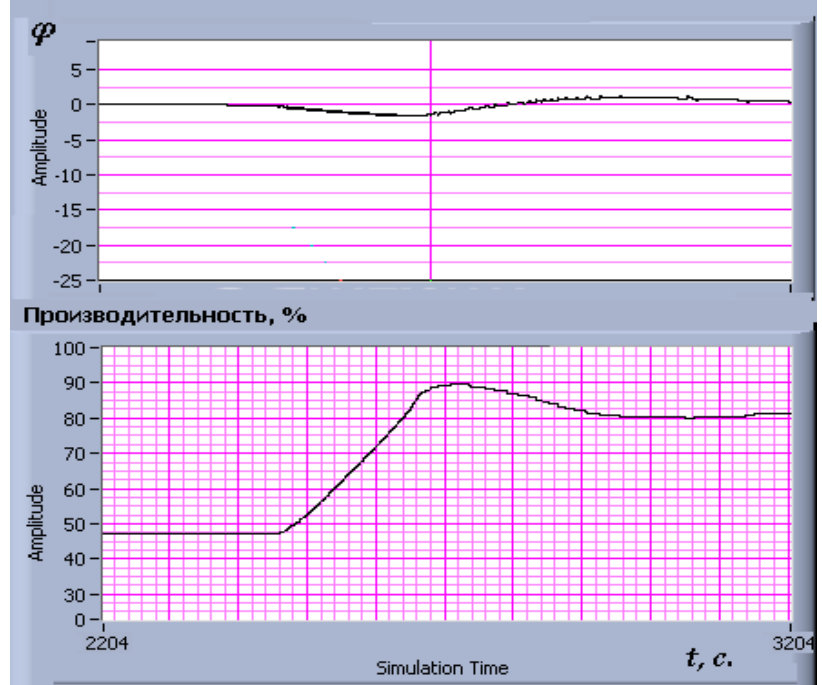

б)

Рис.2 - Графики переходных процессов по производительности турбокомпрессора и по отклонению от соотношения (невязки) при типовой САУ с максимальной производительностью привода системы охлаждения конденсатора а) и при координирующей САУ б)

\section{Выводы}

Исходя из анализа переходных процессов, полученных в результате моделирования, установлено, что качество регулирования производительности холодильного турбокомпрессора при различных САУ практически одинаковое. Однако, предложенная система, построенная на принципе координирующего управления, имеет ряд достоинств. Собственно предложенная система использует известную информацию о траектории дрейфа экстремума, представленную в виде соотношения параметров. При этом, данное соотношение параметров, для энергоэффективного функционирования холодильной установки, обеспечивается как при переходных, так и при стационарных режимах, а работа автоматического оптимизатора в системе сводится лишь только для проверки или корректировки известной информации о траектории дрейфа экстремума. Данные достоинства обусловливают принципиальную пригодность системы для практического применения.

\section{Литература}

1. Холодильные машины: учебник для студентов технических втузов / [Кошкин Н.Н., Сакун И.А., Бамбушек Е.М. и др.]; под общ. ред. проф. И.А., Сакуна / - Л.: Изд-во «Машиностроение», 1985. - 261 с.;

2. Бухарин Н.Н., Евсафьев В.Л., Ковалкин И.К. Совершенствование системы регулирования холодильного центробежного компрессора //Холодильная техника. - 1992. - №9 - С. 7-9.;

3. Сухомлинов И.Я., Савельева И.Ю., Головин М.В. Исследование методов регулирования параметров холодильных машин с центробежными компрессорами // Химическое и нефтяное машиностроение. 1995. - №11 - С. 29-36.;

4. Сухомлинов И.Я., Головин М.В., Славуцкий Д.Л. Регулирование характеристик холодильного центробежного компрессора с изменяемой частотой вращения привода (ОАО «ВНИИхолодмаш-Холдинг», г. Москва) // Химическое и нефтяное машиностроение. - 2007. - №7 - С. 29-36.;

5. Прохоров А.В., Онучин М.Ф. Компрессорные машины с микропроцессорными системами управления, контроля и защиты // Химическое и нефтяное машиностроение. - 1993. - №3 - С. 20-23.;

6. Самонастраивающиеся системы автоматического управления // Справочник по автоматизированному электроприводу / Под ред. В.А. Елисеева и А.В. Шинянского. - М. : Энергоатомиздат, 1983. - С. 362-365.;

7. Гурский А.А. Денисенко В.А., Гончаренко А.Е. Системы автоматического регулирования производительности холодильных центробежных компрессоров // Холодильная техника и технология. Одесса: ОНАХТ, № 5, 2013. - С. 72-76.;

8. Гурський О.О.Автоматизація холодильної турбокомпресорної установки на базі системи координуючого керування: автореферат дис. кандидата тех. наук: 05. 13. 07. - Одесса., 2012. - 22 с.; 


\title{
$\underline{4}$ ТЕХНІЧНІ ЗАСОБИ ТА ІНФОРМАЦІЙНІ ТЕХНОЛОГІЇ В СИСТЕМАХ УПРАВЛІННЯ
}

9. Бойчук Л.М. Синтез координирующих систем автоматического управления - М. : Изд-во «Энергоатомиздат». - 1991. - 160 с.;

10. Чистяков Ф.М. Холодильные турбоагрегаты. - М.: Изд-во «Машиностроение», 1967. - 287 с.;

11. Гурский А.А., Денисенко В.А., Гончаренко А.Е. Невязка по соотношению параметров в системе управления как показатель функционирования турбокомпрессорной установки // Холодильная техника и технология. - Одесса: ОНАХТ, №4 (150), 2014. - С. 58-63.

\section{References}

1. Kholodyl'nыe mashynы: uchebnyk dlya studentov tekhnycheskykh vtuzov / [Koshkyn N.N., Sakun Y.A., Bambushek E.M. y dr.]; pod obshch. red. prof. Y.A., Sakuna / - L.: Yzd-vo «Mashynostroenye», 1985. - 261 s.;

2. Bukharyn N.N., Evsaf'ev V.L., Kovalkyn Y.K. Sovershenstvovanye systemy rehulyrovanyya kholodyl'noho tsentrobezhnoho kompressora //Kholodyl'naya tekhnyka. - 1992. - №9 - S. 7- 9.;

3. Sukhomlynov Y.Ya., Savel'eva Y.Yu., Holovyn M.V. Yssledovanye metodov rehulyrovanyya parametrov kholodyl'nykh mashyn s tsentrobezhnymy kompressoramy // Khymycheskoe y neftyanoe mashynostroenye. 1995. - №11 - S. 29-36;

4. Sukhomlynov Y.Ya., Holovyn M.V., Slavutskyy D.L. Rehulyrovanye kharakterystyk kholodyl'noho tsentrobezhnoho kompressora s yzmenyaemoy chastotoy vrashchenyya pryvoda (OAO «VNYYkholodmashKholdynh», h. Moskva) // Khymycheskoe y neftyanoe mashynostroenye. - 2007. - №7- S. 29-36.;

5. Prokhorov A.V., Onuchyn M.F. Kompressornye mashyny s mykroprotsessornymy systemamy upravlenyya, kontrolya y zashchyty // Khymycheskoe y neftyanoe mashynostroenye. - 1993. - №3 - S. 20-23;

6. Samonastrayvayushchyesya systemy avtomatycheskoho upravlenyya // Spravochnyk po avtomatyzyrovannomu elektropryvodu / Pod red. V.A. Elyseeva y A.V. Shynyanskoho. - M. : Enerhoatomyzdat, - 1983. - S. 362 - 365 ;

7. Hurskyy A.A., Denysenko V.A., Honcharenko A.E. Systemy avtomatycheskoho rehulyrovanyya proyzvodytel'nosty kholodyl'nykh tsentrobezhnykh kompressorov // Kholodyl'naya tekhnyka y tekhnolohyya. Odessa: ONAKhT, №5, 2013. - S. 72-76;

8. Hurskyy O.O. Avtomatyzatsiya kholodyl'noyi turbokompresornoyi ustanovky na bazi systemy koordynuyuchoho keruvannya: avtoreferat dys. kandydata tekh. nauk: 05. 13. 07. - Odessa., 2012. - $22 \mathrm{~s}$.

9. Boychuk L.M. Syntez koordynyruyushchykh system avtomatycheskoho upravlenyya M. : Yzd-vo «Enerhoatomyzdat» - 1991. - 160 s.;

10. Chystyakov F.M. Kholodyl'nye turboahrehaty. - M.: Yzd-vo «Mashynostroenye», 1967. - 287 s.;

11. Hurskyy A.A., Denysenko V.A., Honcharenko A.E. Nevyazka po sootnoshenyyu parametrov v systeme upravlenyya kak pokazatel funktsyonyrovanyya turbokompressornoy ustanovky // Kholodyl'naya tekhnyka y tekhnolohyya. - Odessa: ONAKhT, №4 (150), 2014. - S. 58-63.

\section{AUTOMATION OF PLC PROGRAMMING WHEN IMPLEMENTING ALGORITHMS OF GUARANTEEING CONTROL}

\author{
Levinskyi M.V. ${ }^{1}$, Stepanov M.T. ${ }^{1}$, Khobin V.A. ${ }^{1}$ \\ ${ }^{1}$ Odessa National Academy of Food Technologies, Odessa \\ E-mail: MaxLevinskyi@gmail.com
}

Copyright (C 2014 by author and the journal "Automation technological and business - processes". This work is licensed under the Creative Commons Attribution International License (CC BY). http://creativecommons.org/licenses/by/4.0/

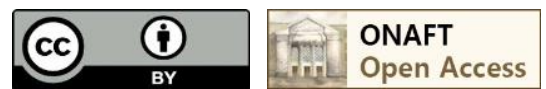

DOI: $10.15673 / 2312-3125$. 